\title{
High Performance Enhancement-Mode AlGaN/GaN MIS-HEMT with Selective Fluorine Treatment
}

\author{
Chao Yang, Jiayun Xiong, Jie Wei, Junfeng Wu, Bo Zhang, and Xiaorong Luo \\ University of Electronic Science and Technology of China, Chengdu 610054, China \\ Correspondence should be addressed to Xiaorong Luo; xrluo@uestc.edu.cn
}

Received 26 February 2015; Revised 5 June 2015; Accepted 17 June 2015

Academic Editor: Ashok Chatterjee

Copyright (C) 2015 Chao Yang et al. This is an open access article distributed under the Creative Commons Attribution License, which permits unrestricted use, distribution, and reproduction in any medium, provided the original work is properly cited.

\begin{abstract}
A novel enhancement-mode (E-mode) Metal-Insulator-Semiconductor- (MIS-) HEMT with selective fluorine ion ( $\left.\mathrm{F}^{-}\right)$treatment is proposed and its mechanism is investigated. The HEMT features the Selective $\mathrm{F}^{-}$treatment both in the AlGaN channel region and in the thick passivation layer between the gate and drain (SFCP-MIS-HEMT). First, the $\mathrm{F}^{-}$in the passivation layer not only extends the depletion region and thus enhances the average electric field ( $E$-field) between the gate and drain by the assisted depletion effect but also reduces the $E$-field peak at the gate end, leading to a higher breakdown voltage (BV). Second, in the AlGaN channel region, the $\mathrm{F}^{-}$region realizes the E-mode and the region without $\mathrm{F}^{-}$maintains a high drain current $\left(I_{D}\right)$. Third, MIS structure suppresses the gate leakage current, increasing the gate swing voltage and the BV. Compared with a MIS-HEMT with $\mathrm{F}^{-}$treatment in whole channel (FC-MIS-HEMT), SFCP-MIS-HEMT increases the BV by $46 \%$ and the saturation drain current $\left(I_{D, \text { sat }}\right)$ by $28 \%$.
\end{abstract}

\section{Introduction}

AlGaN/GaN HEMTs are promising candidates for next generation microwave power amplifiers and high-voltage switches owing to their superior properties $[1,2]$. For such applications, a normally off or E-mode operation is desired, either for fail-safe operation or for simple gate drive circuit design. However, most AlGaN/GaN HEMTs show normally on operation for the high-density two-dimensional electron gas (2DEG). Normally off operation on AlGaN/GaN heterostructures has been obtained with several approaches, such as recessed gate structures [3], PN junction gate structures [4], thinned AlGaN barrier [5], InGaN cap layer [6], and $\mathrm{F}^{-}$treatment [7]. $\mathrm{F}^{-}$treatment in the AlGaN layer is used to realize normally off HEMT [8] but the gate leakage during on-state operation strongly limits the device performance, especially when high threshold voltage $\left(V_{\mathrm{TH}}\right)$ and high current densities are required. By inserting a gate dielectric layer between the gate metal and the semiconductor, $\mathrm{AlGaN} / \mathrm{GaN}$ MIS-HEMTs are expected to address the issues associated with the Schottky gate. The gate insulator material includes $\mathrm{SiO}_{2}$ [9], $\mathrm{Si}_{3} \mathrm{~N}_{4}, \mathrm{TaO}_{x} \mathrm{~N}_{y}$ [10], and $\mathrm{HfO}_{2}$ [11].

The fluoride ions implanted into the $\mathrm{AlGaN}$ barrier layer are also employed to improve the BV [12]. However, $\mathrm{F}^{-}$ treatment in the thin AlGaN barrier induces damage to the
$\mathrm{AlGaN} / \mathrm{GaN}$ heterojunction, and the $\mathrm{F}^{-}$easily gets into the channel and degrades the mobility of the 2DEG. Thus, the forward performance and reliability will be seriously affected.

In this paper, a novel E-mode MIS-HEMT with selective $\mathrm{F}^{-}$treatment both in the channel and in the passivation dielectric (SFCP-MIS-HEMT) is studied. The physical mechanisms of the $\mathrm{F}^{-}$on the device performance are investigated by the theoretical and simulated studies. The physical mechanism is analyzed based on 2D Sentaurus TCAD from Synopsys. The results indicate that the SFCP-MIS-HEMT can significantly improve the $\mathrm{BV}$ and the $I_{D \text {,sat }}$ simultaneously.

\section{Structure and Mechanism}

Figure 1 is the schematic cross section of the proposed SFCPMIS-HEMT. It features the selective $\mathrm{F}^{-}$treatment both in the AlGaN under the gate and in the passivation dielectric between the gate and the drain. $Q_{1}$ and $L_{1}$ represent the $\mathrm{F}^{-}$ concentration and length of the $\mathrm{F}^{-}$treatment region in the AlGaN layer, respectively; $Q_{2}$ and $L_{2}$ are the $\mathrm{F}^{-}$concentration and lateral length of $\mathrm{F}^{-}$treatment in the passivation layer, respectively. When $L_{2}=0$ and $L_{1}<L_{g}$, the structure is selective $\mathrm{F}^{-}$treated in channel (SFC-MIS-HEMT). When $L_{2}=0$ and $L_{1}=L_{g}$, the structure is $\mathrm{F}^{-}$treated in the whole 


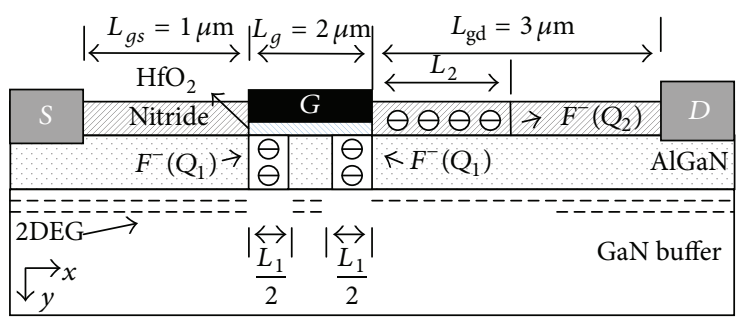

FIGURE 1: Schematic cross section of the proposed SFCP-MIS-HEMT.

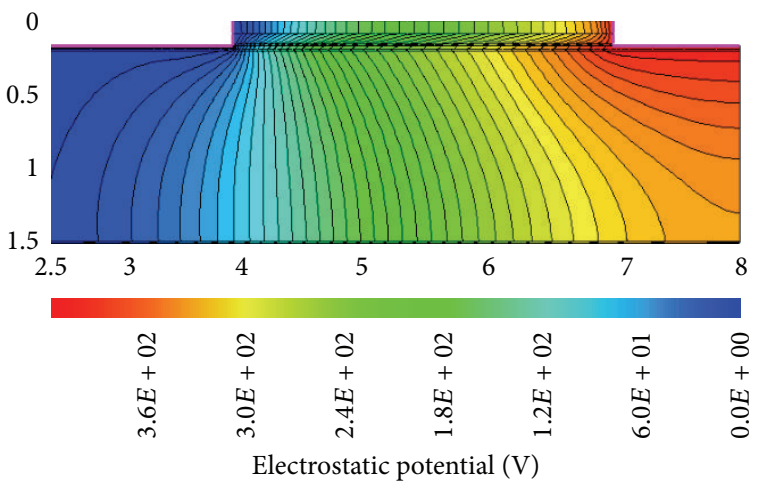

(a) SFCP-MIS-HEMT

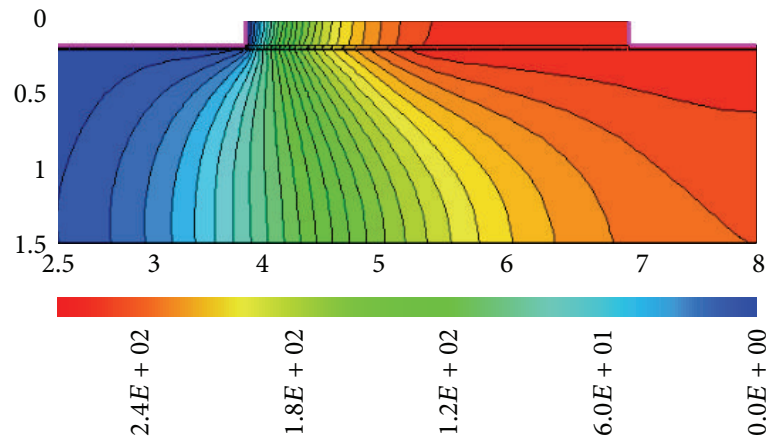

Electrostatic potential (V)

(b) SFC-MIS-HEMT

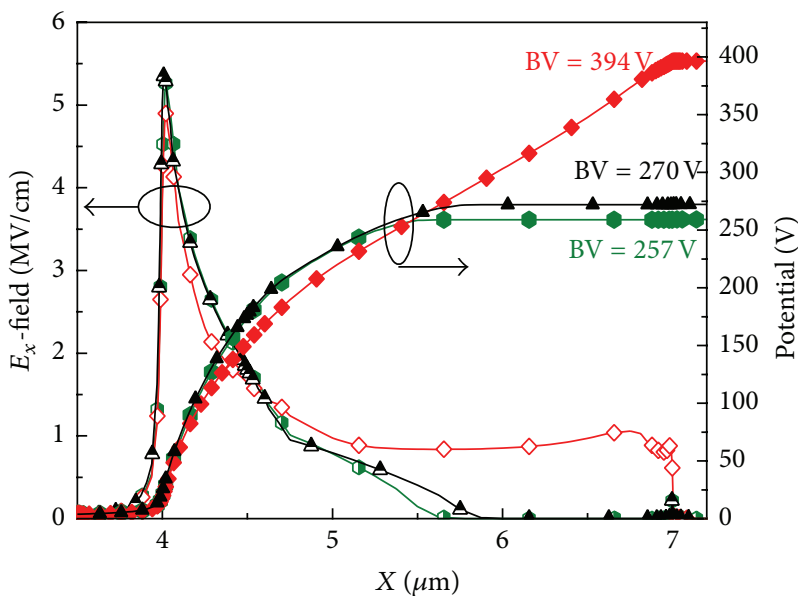

-1- SFC-MIS-HEMT

$\checkmark$ SFCP-MIS-HEMT

$\triangle$ FC-MIS-HEMT

(d)

Figure 2: (a)-(c) Equipotential contours distribution at off-state breakdown of three MIS-HEMTs (10 V/contour); (d) X-component of the surface $E$-field $\left(E_{x}\right)$ and the surface potential distributions at AlGaN/GaN interface.

channel (FC-MIS-HEMT). Table 1 shows abbreviations and parameters of the three different HEMTs in this paper. The $\mathrm{F}^{-}$in the AlGaN under the gate is used in the three types of HEMTs to realize the E-mode. As $\mathrm{F}^{-}$in the passivation layer are immobile negative charges, electrons are depleted from the $2 \mathrm{DEG}$ channel in the implanted region of $\mathrm{F}^{-}$due to the repulsive force. The passivation layer implanted by $\mathrm{F}^{-}$ acts like the field plate. The $\mathrm{F}^{-}$in the passivation layer not only enhances the $E$-field at the drain side by the assisted depletion but also reduces the $E$-field peak at the gate end, leading to a higher BV, with a little effect on the $I_{D \text {,sat }}$. All the three HEMTs are featured by $L_{g}=2 \mu \mathrm{m}$ and a gate-drain distance $L_{\mathrm{gd}}=3 \mu \mathrm{m}$. The HEMTs also consist of a $1.5 \mu \mathrm{m}$ thick GaN layer, a $25 \mathrm{~nm}$ thick AlGaN layer, and $200 \mathrm{~nm}$ thick $\mathrm{Si}_{3} \mathrm{~N}_{4}$ passivation on the AlGaN. The $x$ and $y$ directions are given.

Figure 2 shows the 2D equipotential lines, the surface $E$ field, and potential distribution of the SFCP-, SFC-, and FCMIS-HEMT at off-state breakdown. Comparing Figure 2(a) with Figures 2(b) and 2(c), the $\mathrm{F}^{-}$in the passivation layer 


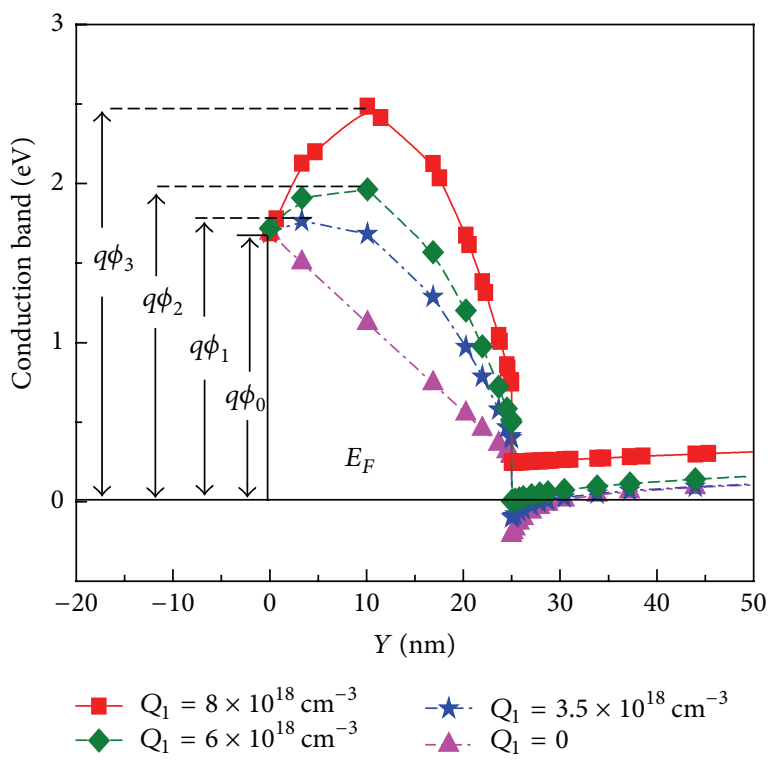

(a)

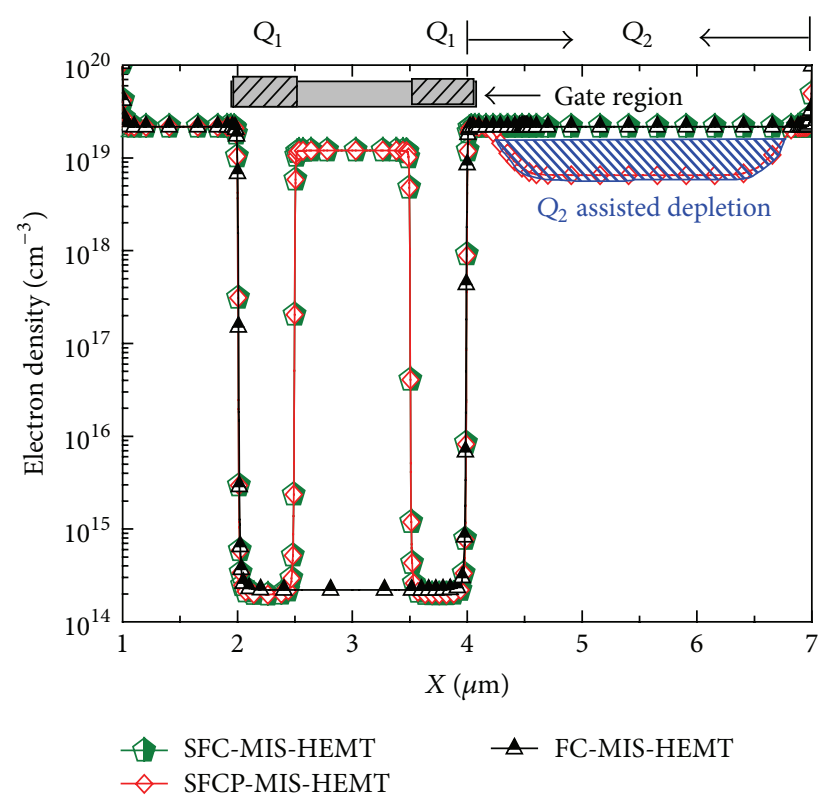

(b)

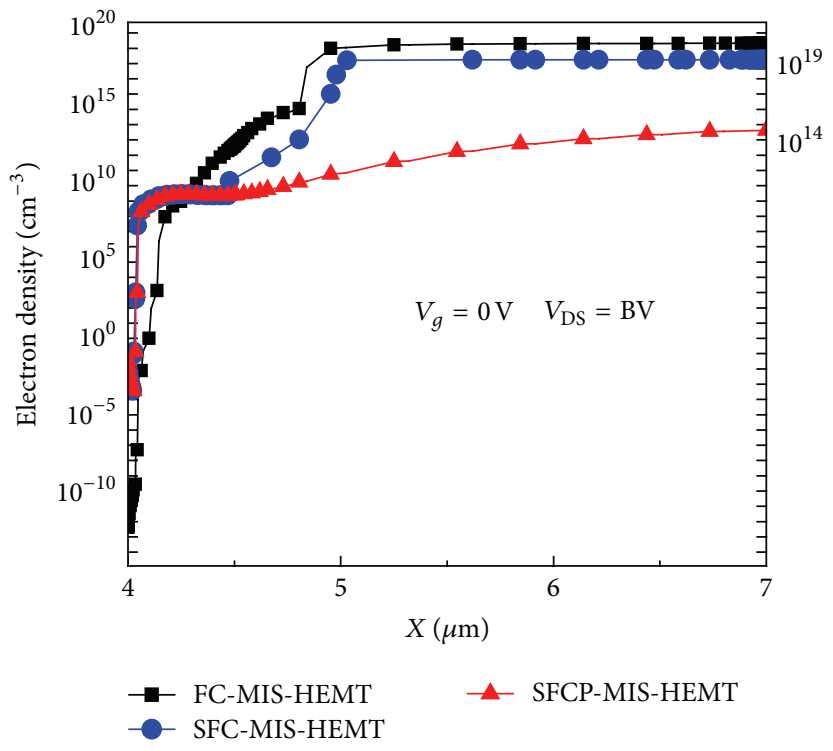

(c)

FIGURE 3: (a) Influence of $Q_{1}$ on conduction band; (b) 2DEG density of the three different MIS-HEMTs at on state; (c) 2DEG density of the three different MIS-HEMTs in the drift region at off state.

TABLE 1: Abbreviations and parameters of three HEMTs.

\begin{tabular}{lcc}
\hline Structure name & $L_{1}$ & $L_{2}$ \\
\hline SFCP-MIS-HEMT & $L_{1}<L_{g}$ & $L_{2} \neq 0$ \\
SFC-MIS-HEMT & $L_{1}<L_{g}$ & $L_{2}=0$ \\
FC-MIS-HEMT & $L_{1}=L_{g}$ & $L_{2}=0$ \\
\hline
\end{tabular}

extends the depletion region and makes the potential lines more uniform between the gate and the drain. In Figure 2(d), owing to the modulation effect of the $\mathrm{F}^{-}$in the passivation layer, the $x$-component of surface $(y=0.226 \mu \mathrm{m}) E$ field $\left(E_{x}\right)$ distributions of the SFCP-MIS-HEMT is more uniform and higher than that of the FC-MIS-HEMT and SFC-MIS-HEMT, and the peak electric field at the gate edge is decreased, leading to a higher BV. The surface potential almost linearly increases from the gate to the drain. However, because the buffer layer leakage causes the device premature breakdown, the peak $E$-field around the drain edge of SFCPMIS-HEMT cannot continue improving.

Figure 3(a) shows the influence of $Q_{1}$ on conduction band under the gate. The $Q_{1}$ elevates the conduction band above the Fermi level $\left(E_{F}\right)$ at $Q_{1} \geq 6 \times 10^{18} \mathrm{~cm}^{-3}$, realizing the Emode. Figures $3(\mathrm{~b})$ and $3(\mathrm{c})$ show the $2 \mathrm{DEG}$ density at the on state and off state. In Figure 3(b), for the SFCP-MIS-HEMT, the $Q_{1}$ region depletes the 2DEG and realizes the E-mode, 

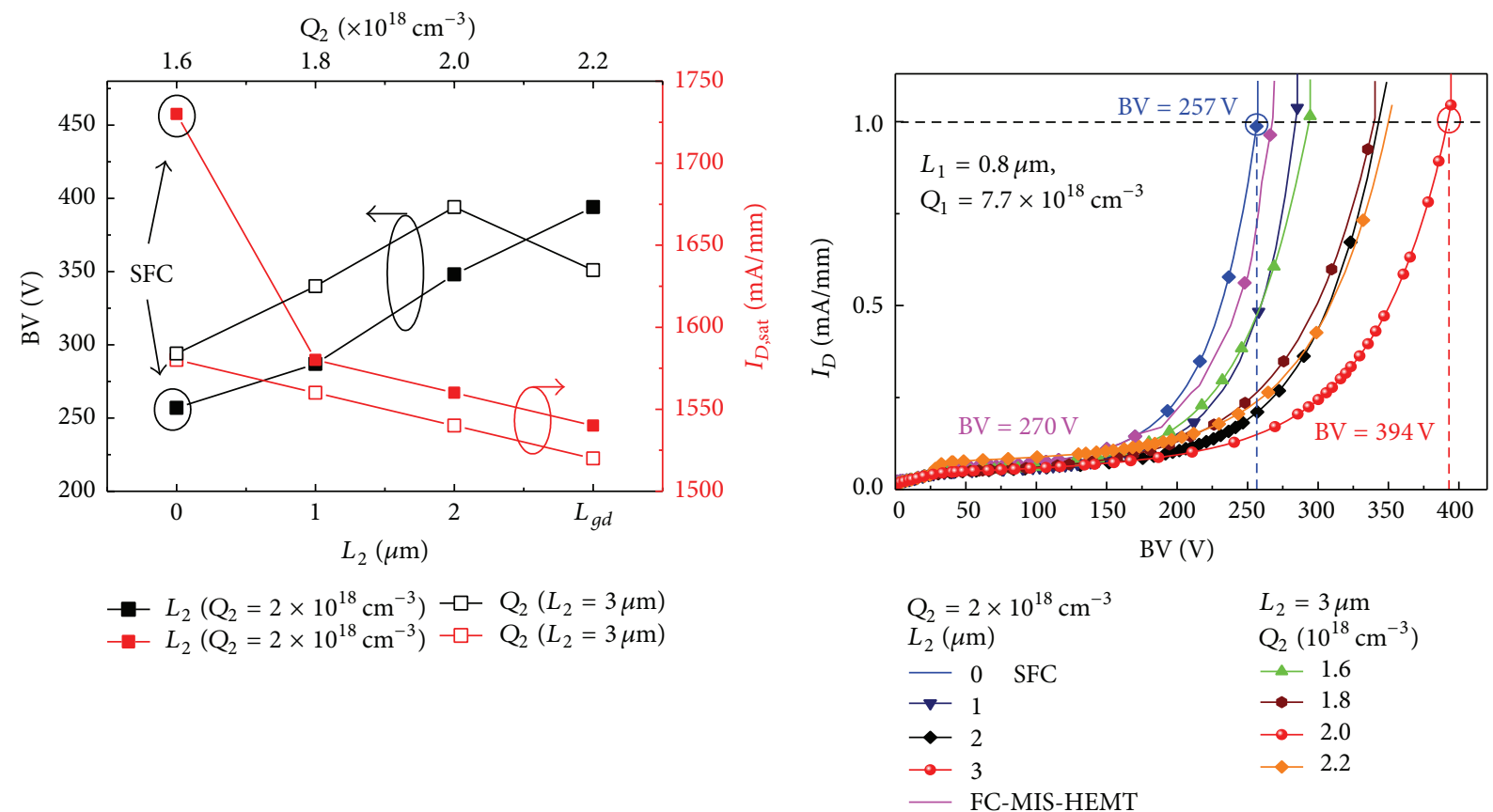

(a)

(b)

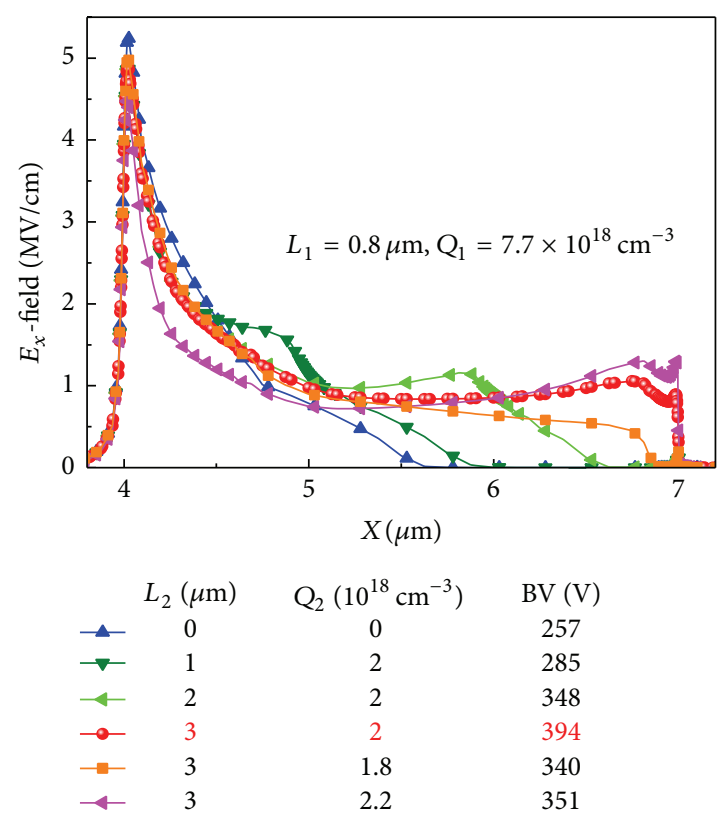

(c)

Figure 4: (a) Influences of $L_{2}$ and $Q_{2}$ on BV and $I_{D \text {,sat }}$; (b) $I-V$ curves for the different $L_{2}$ and $Q_{2}$ values, where the BV is defined as the source-drain voltage at $I_{D}=1 \mathrm{~mA} / \mathrm{mm}$ and $V_{g}=0 \mathrm{~V}$; (c) $E_{x}$-field at AlGaN/GaN interface.

while the region without $\mathrm{F}^{-}$maintains a high $2 \mathrm{DEG}$ density under the gate so as to obtain a high on-state $I_{D}$. The $\mathrm{F}^{-}$in the passivation layer has a little effect on the $2 \mathrm{DEG}$ density at on state. At the off-state $V_{\mathrm{DS}}=\mathrm{BV}$, the $2 \mathrm{DEG}$ density in the drift region of the FC-MIS-HEMT and SFC-MISHEMT keeps a high value, providing a leakage path. With the assisted depletion caused by the $\mathrm{F}^{-}$in the passivation layer, the 2DEG density of the SFCP-MIS-HEMT in the drift region is decreased by five orders of magnitude as shown in
Figure 3(c). Therefore, the buffer layer leakage current from the drain to the source is depressed and a higher BV is achieved.

\section{Results and Discussion}

The influence of $L_{2}$ and $Q_{2}$ on the off-state BV and $I_{D \text {,sat }}$ are shown in Figure 4(a). The BV increases with the increasing $L_{2}$ owing to the spreading depletion region. As the $Q_{2}$ increases, 


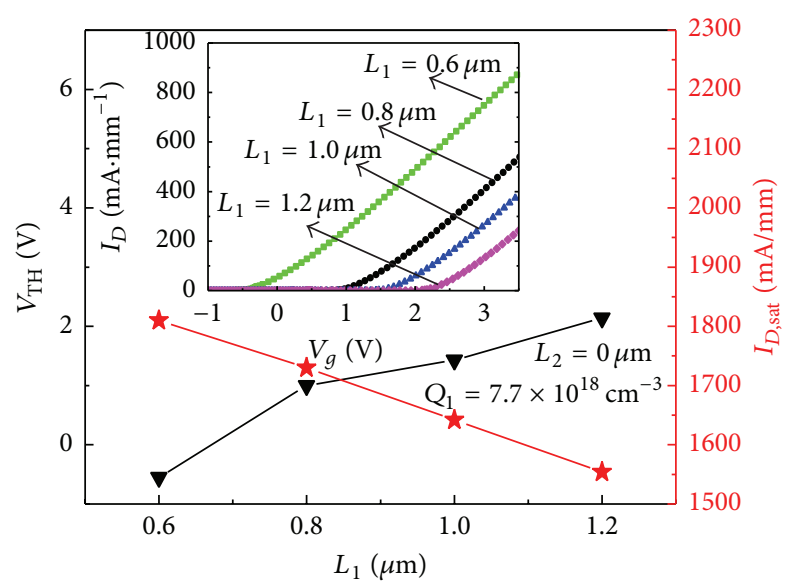

(a)

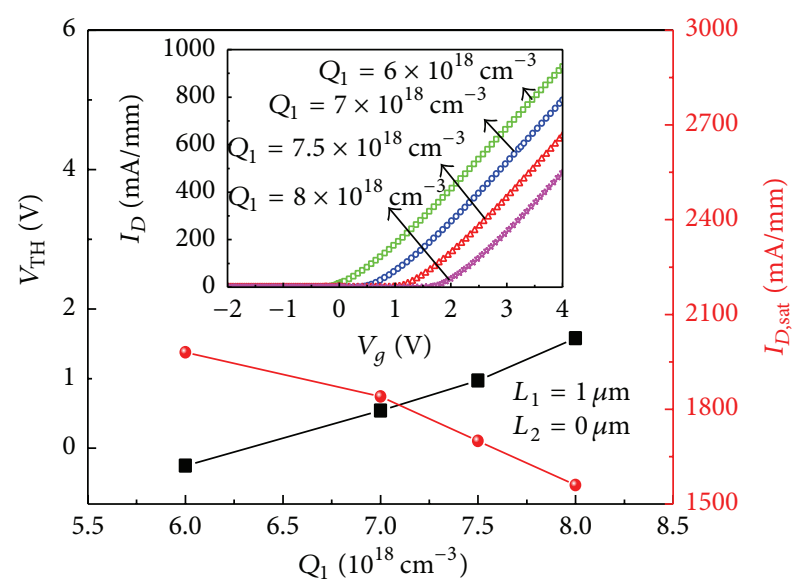

(b)

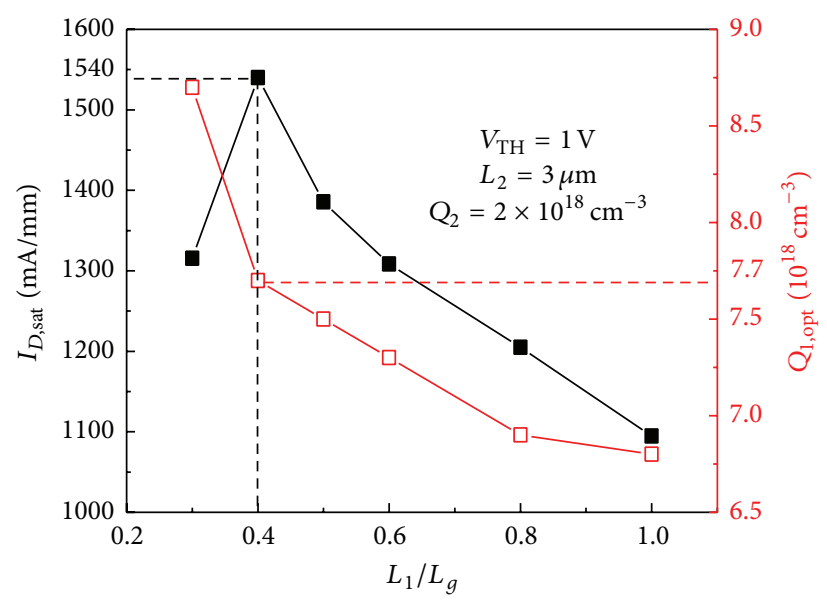

(c)

Figure 5: (a) Dependence of the $V_{\mathrm{TH}}$ and $I_{D, \text { sat }}$ on the $L_{1}$; (b) dependence of the $V_{\mathrm{TH}}$ and $I_{D, \text { sat }}$ on the $Q_{1}$; (c) influence of the $L_{1}$ on the $I_{D, \text { sat }}$.

the BV first increases and then decreases because the $E_{x}$-field at drain side steadily increases while the $E_{x}$-field at gate side begins to decrease at $Q_{2}=2.2 \times 10^{18} \mathrm{~cm}^{-3}$. With the increase in the $Q_{2}$ and $L_{2}$, the $I_{D \text {,sat }}$ slightly decreases due to the enhanced assisted depletion on the 2DEG. The $I-V$ curves for the SFCP-MIS-HEMT with different $Q_{2}$ and $L_{2}$ are compared at $V_{g}=0 \mathrm{~V}$ in Figure 4(b). In Figure 4(b), the SFCP-MISHEMT can realize the highest BV in these three HEMTs. First, the depletion width and the E-field strength in the drift are enhanced due to the assisted depletion caused by the $\mathrm{F}^{-}$ in the passivation layer $\left(Q_{2}\right)$. Second, the peak $E$-field around the gate edge is decreased by $Q_{2}$, avoiding the premature breakdown herein (see Figures 4(c) and 2(d)). Figure 4(b) also shows that the $\mathrm{BV}$ increases with the increasing $L_{2}$ in the range of $0-3 \mu \mathrm{m}$ owing to the spreading depletion region between the gate and the drain. $Q_{2}$ can increase the $E$-field strength near the drain and thus improve the BV while the too high $Q_{2}$ (e.g., $Q_{2}=2.2 \times 10^{18} \mathrm{~cm}^{-3}$ ) reduces the $E$ field strength near the gate and leads to a decrease in the BV. Note that the premature breakdown caused by the BUFFER layer leakage prevents the $E$-field peak at the drain end from further increasing with the increasing $L_{2}$ and $Q_{2}$. Figure 4(c) illustrates that the $E_{x}$-field distributions of the SFCP-MISHEMT are more uniform and higher than those of the FCMIS-HEMT and the SFC-MIS-HEMT.

Figure 5 indicates the dependence of the $V_{\mathrm{TH}}$ and $I_{D \text {,sat }}$ on the $Q_{1}$ and $L_{1}\left(V_{\mathrm{TH}}=1 \mathrm{~V}\right)$. Figure 5(a) shows that the $V_{\mathrm{TH}}$ increases with the increasing $L_{1}$ at $Q_{1}=7.7 \times 10^{18} \mathrm{~cm}^{-3}$ and $L_{2}=0 \mu \mathrm{m}$. However, with the increases in the $L_{1}$, the $I_{D \text {,sat }}$ obviously decreases due to the enhanced depletion on the 2DEG. In Figure 5(b), the $V_{\mathrm{TH}}$ increases with the increasing $Q_{1}$ at $L_{1}=0.5 \mu \mathrm{m}$ and $L_{2}=0 \mu \mathrm{m}$. The inset in Figures 5(a) and 5(b) shows the transfer characteristic curves as a function of $Q_{1}$ and $L_{1}$. To make sure $V_{\mathrm{TH}}=1 \mathrm{~V}$, the optimized $Q_{1}\left(Q_{1, \text { opt }}\right)$ decreases with the increasing $L_{1}$. When the $L_{1}$ is too small, the corresponding $Q_{1 \text {,opt }}$ is extremely high (e.g., $L_{1}=0.6 \mu \mathrm{m}$ and $Q_{1}=8.7 \times 10^{18} \mathrm{~cm}^{-3}$ ) and the $2 \mathrm{DEG}$ density and mobility are very low, leading to a sharp decrease in $I_{D \text {,sat }}$ as shown in Figure 5(c). Finally, the minimum value of $L_{1}$ is $0.8 \mu \mathrm{m}$.

The transfer characteristic and output characteristic are compared for the SFCP-, SFC-, and FC-MIS-HEMT in Figure 6. Three HEMTs have the same threshold voltage of $V_{\mathrm{TH}}=1 \mathrm{~V}$. In Figure $6(\mathrm{a})$, the $I_{D \text {,sat }}$ of the SFCP-, 


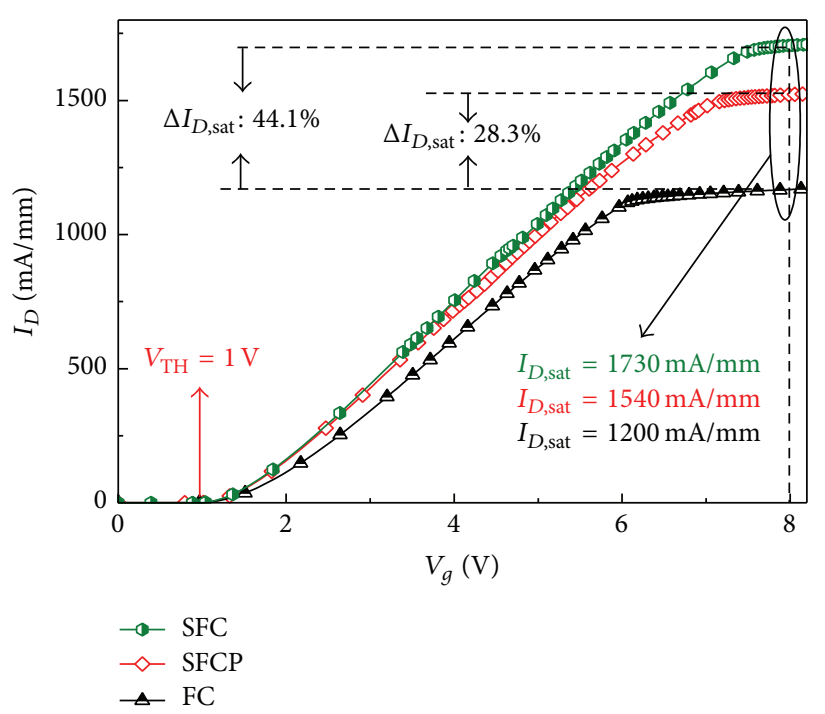

(a)

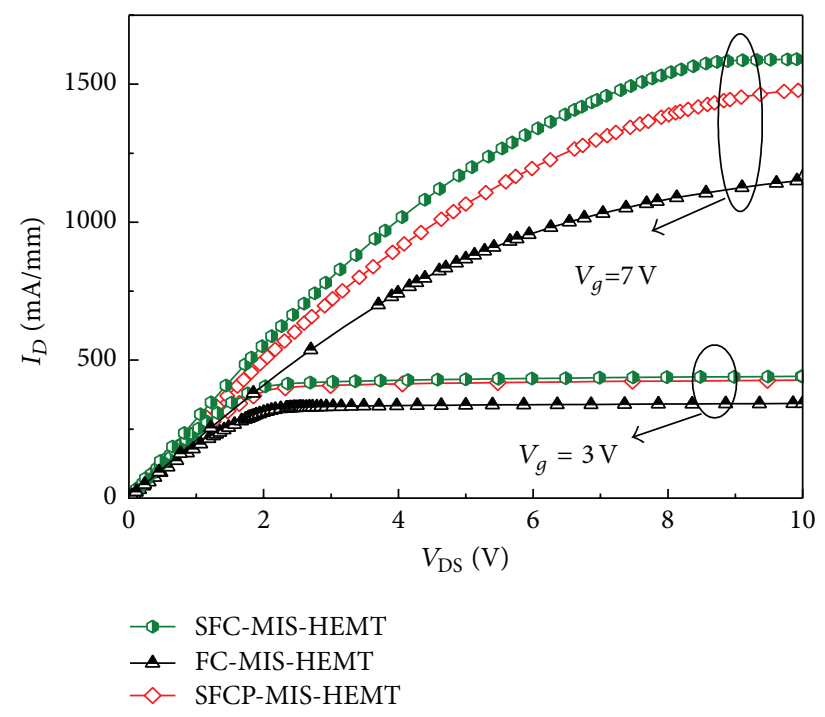

(b)

FIgURE 6: (a) Transfer characteristic curves at $V_{\mathrm{DS}}=10 \mathrm{~V}$; (b) output characteristic curves for the three structures.

SFC-, and FC-MIS-HEMT is $1540 \mathrm{~mA} / \mathrm{mm}, 1730 \mathrm{~mA} / \mathrm{mm}$, and $1200 \mathrm{~mA} / \mathrm{mm}$, respectively. Compared with the SFCMIS-HEMT, SFCP-MIS-HEMT has a small decrease in the $I_{D \text {,sat }}$ because $\mathrm{F}^{-}$in the passivation layer causes the assisted depletion of the 2DEG in the drift region at large drain bias. In Figure 6(b), the drain current of the FC-MIS-HEMT is the lowest and that of the other two HEMTs are almost the same at $V_{g}=3 \mathrm{~V}$. The on resistance $\left(R_{\text {on }}\right)$ mainly consists of the channel resistance $\left(R_{\mathrm{ch}}\right)$ and the drift resistance $\left(R_{d}\right)$. When the $V_{g}$ is small (e.g., $V_{g}=3 \mathrm{~V}$ ), the $R_{\text {ch }}$ accounts for the major part of the $R_{\mathrm{on}}$. The $R_{\mathrm{ch}}$ of the FC-MIS-HEMT is the highest and that of the other two HEMTs is almost the same. As the $V_{g}$ increases, the $R_{d}$ gets more and more important. At $V_{g}=7 \mathrm{~V}$, the $I_{D}$ of the SFCP-MIS-HEMT is smaller than that of the SFC-MIS-HEMT because of a higher $R_{d}$ caused by the assisted depletion effect of the $Q_{2}$ on the 2DEG. The degradation in the forward characteristics is slight nevertheless since the $Q_{2}$ is relatively low and the $\mathrm{F}^{-}$in the passivation layer induces no damage to the $\mathrm{AlGaN} / \mathrm{GaN}$ heterojunction. As a result, an excellent trade-off between the off-state characteristics and the on-state characteristics is achieved.

Figure 7 illustrates the specific on resistance $\left(R_{\mathrm{on}, \mathrm{sp}}\right)$ and off-state BV of the SFCP-MIS-HEMT and recently reported normally off AlGaN/GaN HEMTs, together with the theoretical $\mathrm{Si}, \mathrm{SiC}$, and $\mathrm{GaN}$ limit. It indicates that SFCP-MIS-HEMT has a higher Figure of Merit $\left(\mathrm{FOM}=\mathrm{BV}^{2} / R_{\mathrm{on}, \mathrm{sp}}\right)$. The SFCPMIS-HEMTs exhibit almost a linear $R_{\mathrm{on}, \mathrm{sp}}-\mathrm{BV}$ dependence as shown in Figure 7. The performance of the SFCP-MISHEMTs is better than that of the mentioned AlGaN/GaN HEMTs. It also implies that a higher BV can be expected in the proposed devices with a larger $L_{\text {gd }}$.

\section{Conclusion}

The off-state and on-state performances of the proposed AlGaN/GaN HEMT are improved by selective $\mathrm{F}^{-}$treatment

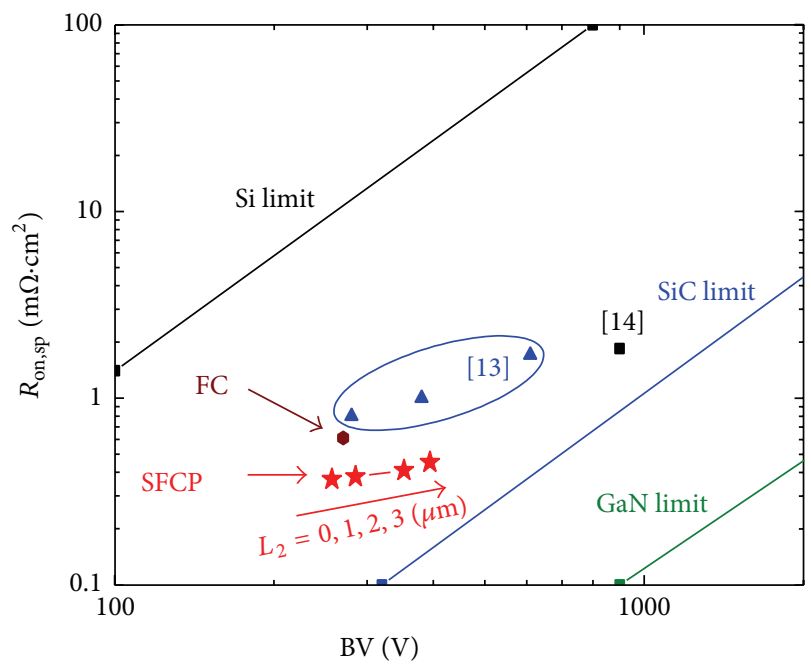

FIGURE 7: $R_{\text {on,sp }}$ and off-state BV values in the SFCP-MIS-HEMT and recently reported normally off AlGaN/GaN HEMTs $[13,14]$.

in both the passivation layer and the AlGaN barrier layer. The $\mathrm{F}^{-}$in the passivation layer not only enhances the electric field near the drain side but also reduces the $E$-field peak at the gate end, leading to a higher breakdown voltage, without the obvious effect on the $I_{D \text {,sat }}$. Moreover, as for as the AlGaN channel region, the $\mathrm{F}^{-}$region realizes the E-mode and the region without $\mathrm{F}^{-}$maintains a high $I_{D}$. The proposed devices possess a better trade-off relationship between $\mathrm{BV}$ and $R_{\mathrm{on}, \mathrm{sp}}$. Therefore, the selective $\mathrm{F}^{-}$treatment technique is available to achieve power devices with the capacity of high voltage, high output power, and low power dissipation.

\section{Conflict of Interests}

The authors declare that there is no conflict of interests regarding the publication of this paper. 


\section{References}

[1] T. P. Chow and R. Tyagi, "Wide bandgap compound semiconductors for superior high-voltage unipolar power devices," IEEE Transactions on Electron Devices, vol. 41, no. 8, pp. 1481-1483, 1994.

[2] O. Ambacher, B. Foutz, J. Smart et al., "Polarization induced effects in AlGaN/GaN heterostructures," Journal of Applied Physics, vol. 87, no. 1, pp. 334-344, 2000.

[3] S. D. Burnham, K. Boutros, P. Hashimoto et al., "Gate-recessed normally-off GaN-on-Si HEMT using a new $\mathrm{O}_{2}-\mathrm{BCl}_{3}$ digital etching technique," Physica Status Solidi C: Current Topics in Solid State Physics, vol. 7, no. 7-8, pp. 2010-2012, 2010.

[4] X. Hu, G. Simin, J. Yang, M. A. Khan, R. Gaska, and M. S. Shur, "Enhancement mode AlGaN/GaN HFET with selectively grown pn junction gate," Electronics Letters, vol. 36, no. 8, pp. 753-754, 2000.

[5] Y. Ohmaki, M. Tanimoto, S. Akamatsu, and T. Mukai, "Enhancement-mode AlGaN/AlN/GaN high electron mobility transistor with low on-state resistance and high breakdown voltage," Japanese Journal of Applied Physics, vol. 45, no. 42-45, pp. L1168-L1170, 2006.

[6] T. Mizutani, M. Ito, S. Kishimoto, and F. Nakamura, "AlGaN/GaN HEMTs with thin InGaN cap layer for normally off operation," IEEE Electron Device Letters, vol. 28, no. 7, pp. 549-551, 2007.

[7] H. Chen, M. Wang, and K. J. Chen, "Self-aligned enhancementmode AIGaN/GaN HEMTs using 25 ke V fluorine ion implantation," in Proceedings of the 68th Device Research Conference (DRC '10), pp. 137-138, June 2010.

[8] Y. Cai and K. J. Chen, "High-performance enhancement-mode AlGaN/GaN HEMTs using fluoride-based plasma treatment," IEEE Electron Device Letters, vol. 26, no. 7, pp. 435-437, 2005.

[9] L. Pang, Y. Lian, D.-S. Kim, J.-H. Lee, and K. Kim, "AlGaN/GaN MOSHEMT with high-quality gate- $\mathrm{SiO}_{2}$ achieved by roomtemperature radio frequency magnetron sputtering," IEEE Transactions on Electron Devices, vol. 59, no. 10, pp. 2650-2655, 2012.

[10] T. Sato, J. Okayasu, M. Takikawa et al., "AlGaN-GaN metalinsulator semiconductor high-electron-mobility transistors with very high-k oxynitride $\mathrm{TaOxNy}$ gate dielectric," IEEE Electron Device Letters, vol. 34, no. 3, pp. 375-377, 2013.

[11] O. Seok, W. Ahn, M.-K. Han, and M.-W. Ha, "High on/off current ratio AlGaN/GaN MOS-HEMTs employing RF-sputtered $\mathrm{HfO}_{2}$ gate insulators," Semiconductor Science and Technology, vol. 28, no. 2, Article ID 025001, 2013.

[12] Y.-S. Kim, J. Lim, O.-G. Seok, and M.-K. Han, "High breakdown voltage AlGaN/GaN HEMT by employing selective fluoride plasma treatment," in Proceedings of the 23rd International Symposium on Power Semiconductor Devices and ICs (ISPSD '11), pp. 251-255, San Diego, Calif, USA, May 2011.

[13] Z. Tang, Q. Jiang, Y. Lu et al., " $600 \mathrm{~V}$ normally off SiNx/AlGaN/GaN MIS-HEMT with large gate swing and low current collapse," IEEE Electron Device Letters, vol. 34, no. 11, pp. 1373-1375, 2013.

[14] M. Wang, Y. Wang, C. Zhang et al., " $900 \mathrm{~V} / 1.6 \mathrm{~m} \Omega \cdot \mathrm{cm}^{2}$ normally-off $\mathrm{Al}_{2} \mathrm{O}_{3}$-GaN MOS-FET on silicon substrate," IEEE Transactions on Electron Devices, vol. 61, no. 6, pp. 2035-2040, 2014. 

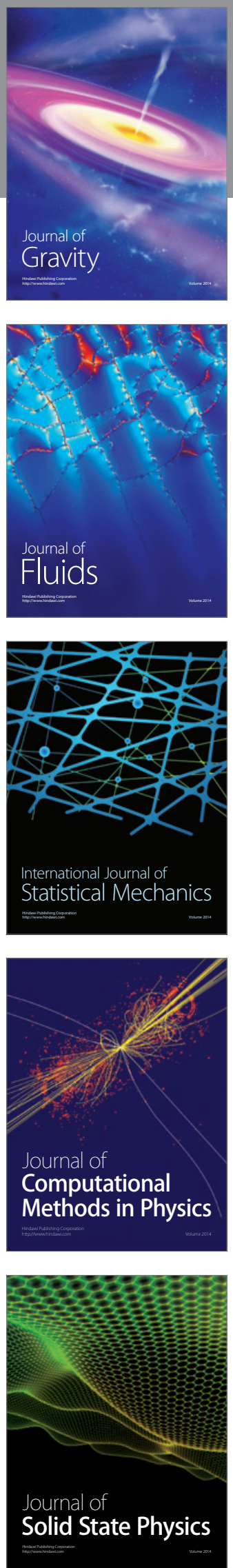

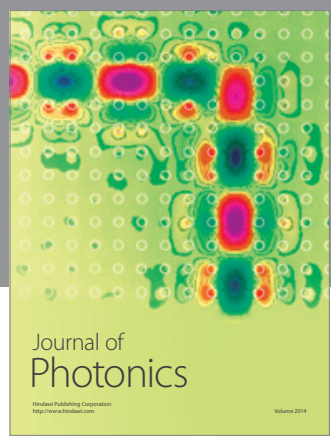

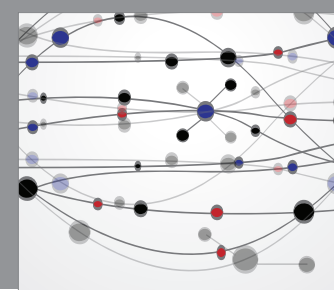

The Scientific World Journal

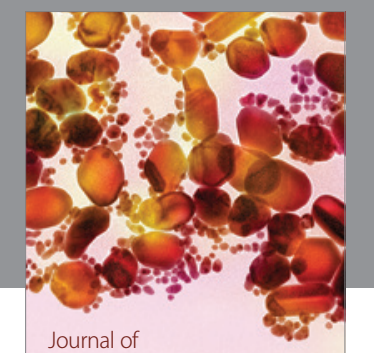

Soft Matter
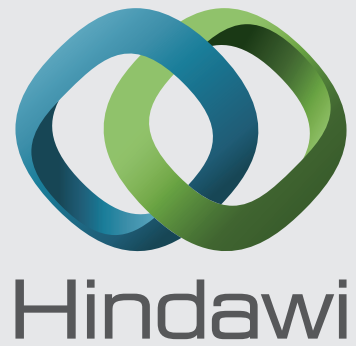

Submit your manuscripts at

http://www.hindawi.com
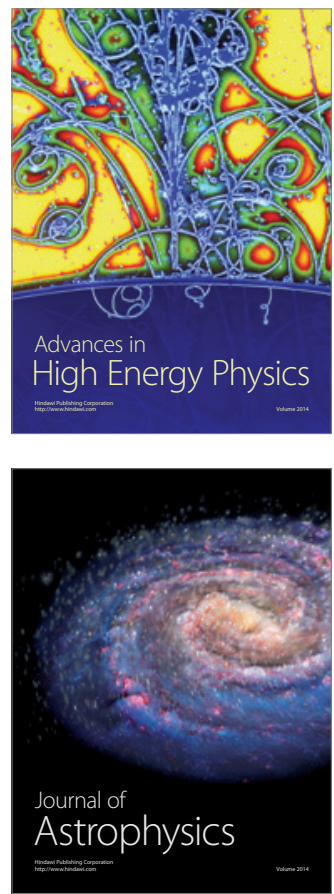
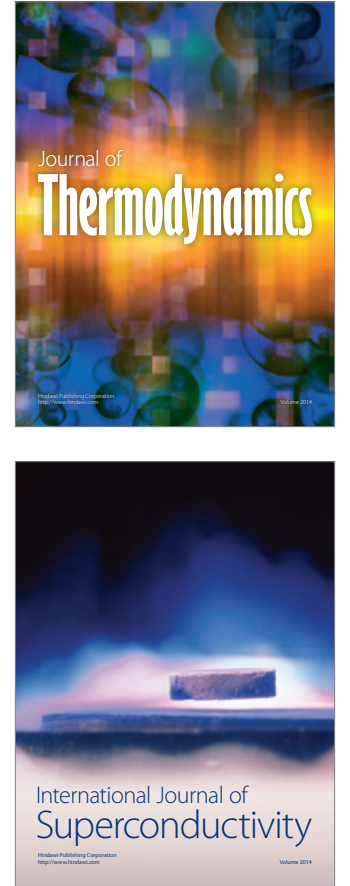
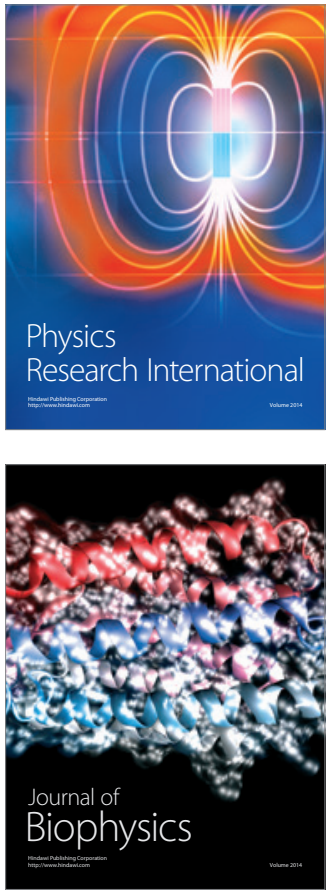
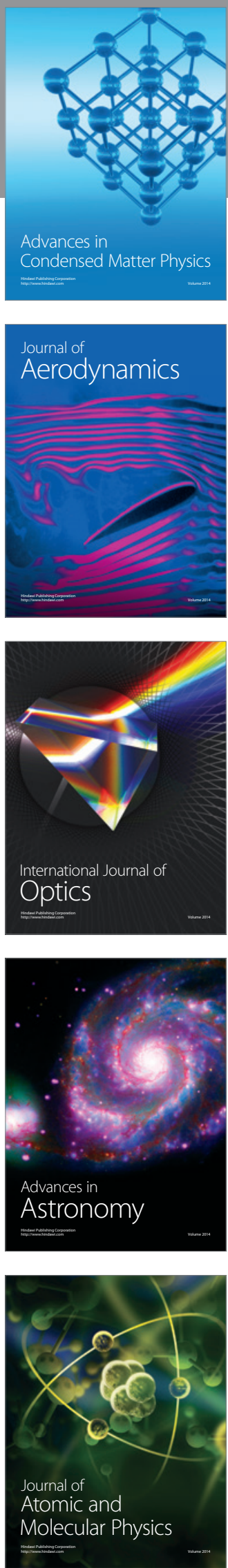